\title{
Anaerobic conversion of biomass upon disintegration in ultrasonic fields of low intensity
}

\author{
S. E. Shcheklein, A. V. Chugunov \& E. V. Arbuzova \\ The Ural Federal University, Russia
}

\begin{abstract}
Disintegration is the destruction of the cell structure to release all its contents. This process facilitates bacteria access to nutrients and it results in a substrate decomposition rate increase. We can use thermal (the brief heating below one hundred degrees temperature at normal pressure or higher one hundred degrees temperature at enhanceable pressure) and chemical (enzymes and catalysts addition, which can positively influence the process speed), biochemical and physical-mechanical methods (the sonication).

Keywords: biogas, biomass, sonication.
\end{abstract}

\section{Introduction}

The purpose of all influencing the substrate processes is the gas yield increase and the fermentation period reduce. In accordance with the AG ZORG data and some other investigators the gas yield after the ultrasonic disintegration increases by 45 percent. But too powerful sonication leads to the bacteria destruction therefore currently used ultrasound low power. Another previous experience is the biomass preliminary sonication Ufa State Oil Technical University was patented (RF patent number 2458868). And also method for preparing to use of liquid manure was patented by Voronezh State Agricultural University (RF patent number 2039028). But above sonication system creators didn't consider the sonication optimum power finding problem. The purpose of our work is the biomass where the humidity is 92 percent sonication optimum determination. 


\section{Experimental investigation}

Experiments were carried out on the "Sapphire" plant that contains transmitters produced by APC International Ltd (USA). The transmitters' power varies from 10 to $150 \mathrm{~kW}$ at a frequency of $44 \mathrm{kHz}$.

\subsection{Experimental stand and sonication regimes}

Figure 1 shows the experimental stand scheme.

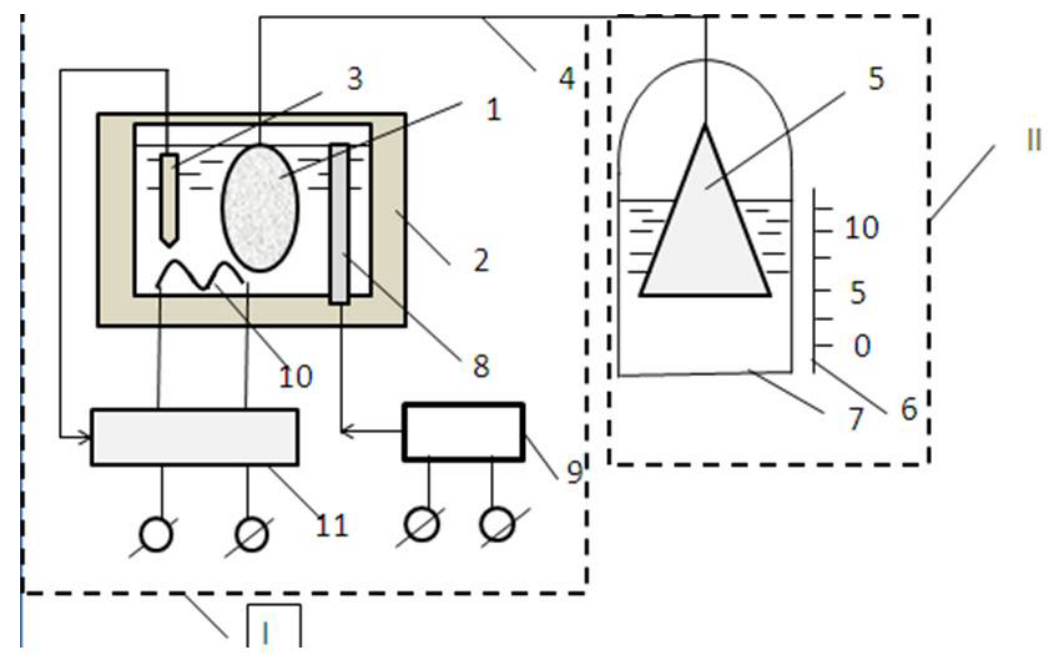

Figure 1: Experimental plant based on the ultrasound chamber "Sapphire". I - ultrasonic camera "Sapphire", II - gasholder, 1 - bioreactor, 2 -thermostat, 3 - temperature sensor, 4 - gas yield, 5 - pop thing, 6 - scale, 7 - water, 8 -ultrasonic transmitter, 9 - ultrasonic generator, 10 - thermostat, 11 - temperature control unit.

Sonication regimes are shown in Table 1.

\subsection{Methodology to evaluate the ultrasound impact}

Sonication dose was calculated as follows:

$$
D=N \cdot t,
$$

$\mathrm{N}$ - sonication power, $\mathrm{W}$;

$t$-sonication time, an hour proportion. 
Table 1: $\quad$ Sonication regimes.

\begin{tabular}{|c|c|c|c|c|c|}
\hline $\begin{array}{c}\text { Investigated } \\
\text { regimes }\end{array}$ & $\begin{array}{c}\text { Sonication } \\
\text { time (min) }\end{array}$ & $\begin{array}{c}\text { Sonication } \\
\text { power (W) }\end{array}$ & $\begin{array}{c}\text { Sonication } \\
\text { dose (Wh) }\end{array}$ & $\begin{array}{c}\text { Sonication } \\
\text { power } \\
\text { density } \\
\left(\mathrm{W} / \mathrm{cm}^{3}\right)\end{array}$ & $\begin{array}{c}\text { Sonication } \\
\text { energy } \\
\text { density } \\
\left(\mathrm{Wh} / \mathrm{cm}^{3}\right)\end{array}$ \\
\hline 1 & 2 & 37.5 & 1.25 & 0.15 & 0.005 \\
\hline 2 & 15 & 37.5 & 9.375 & 0.15 & 0.0375 \\
\hline 3 & 20 & 37.5 & 12.5 & 0.15 & 0.05 \\
\hline 4 & 30 & 37.5 & 18.75 & 0.15 & 0.075 \\
\hline 5 & 2 & 75 & 2.5 & 0.3 & 0.01 \\
\hline 6 & 5 & 75 & 6.25 & 0.3 & 0.025 \\
\hline 7 & 10 & 75 & 12.5 & 0.3 & 0.05 \\
\hline 8 & 20 & 75 & 25 & 0.3 & 0.1 \\
\hline 9 & 30 & 75 & 37.5 & 0.3 & 0.15 \\
\hline 10 & 2 & 150 & 5 & 0.6 & 0.02 \\
\hline 11 & 5 & 150 & 12.5 & 0.6 & 0.05 \\
\hline 12 & 10 & 150 & 25 & 0.6 & 0.1 \\
\hline 13 & 15 & 150 & 37.5 & 0.6 & 0.15 \\
\hline 14 & 30 & 150 & 50 & 0.6 & 0.2 \\
\hline
\end{tabular}

Sonication power density was calculated as:

$$
Y^{\prime}=\frac{N}{V_{\text {субст }}}
$$

$V_{\text {субст }}$ - the substrate being processed volume in the sample.

Sonication energy density was calculated as:

$$
Y^{\prime \prime}=\frac{D}{V_{\text {субст }}}
$$

$\mathrm{D}$ - sonication dose.

\subsection{Sonication dose influence}

Figures 2 and 3 show biogas nonflammable components formation dynamic nature in the initial fermentation stage $\left(\mathrm{CO}_{2}, \mathrm{O}_{2}\right)$ and biogas flammable components $\left(\mathrm{CH}_{4}, \mathrm{H}_{2}\right)$ formation data at the methanogenesis stage. The data analysis shows that the biogas flammable components maximum increase is 34$43 \%$ at the $5-10$ Wh sonication dose. 
1020 Energy Production and Management in the 21st Century, Vol. 2

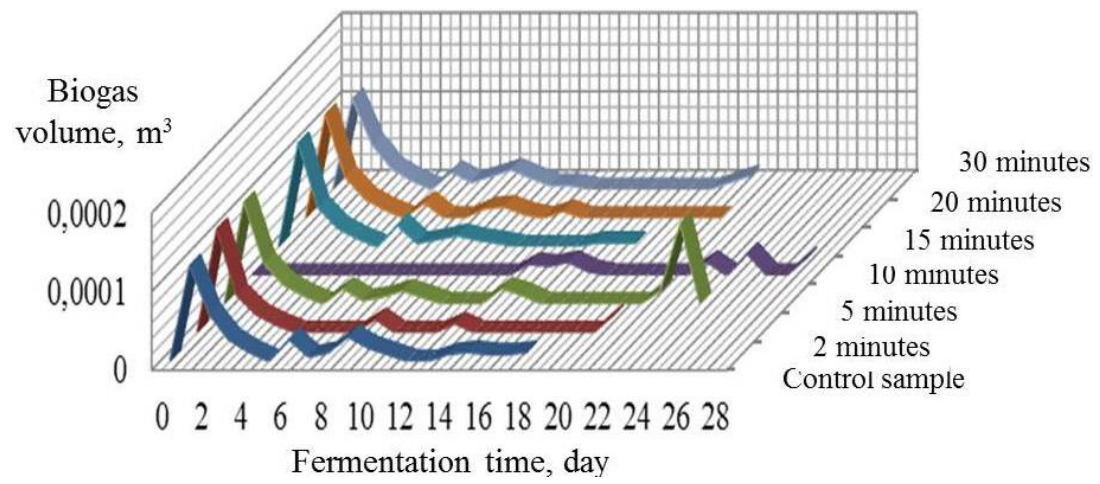

Figure 2: The gas yield in the initial fermentation stage.

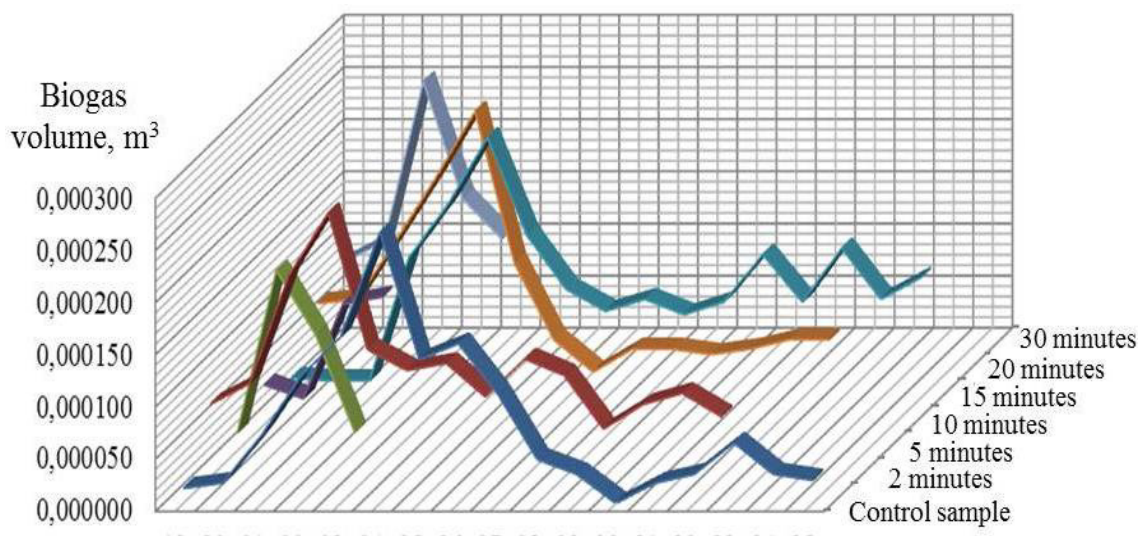

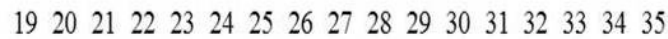

Fermentation time, day

Figure 3: Biogas flammable components yield at the methanogenesis stage. 


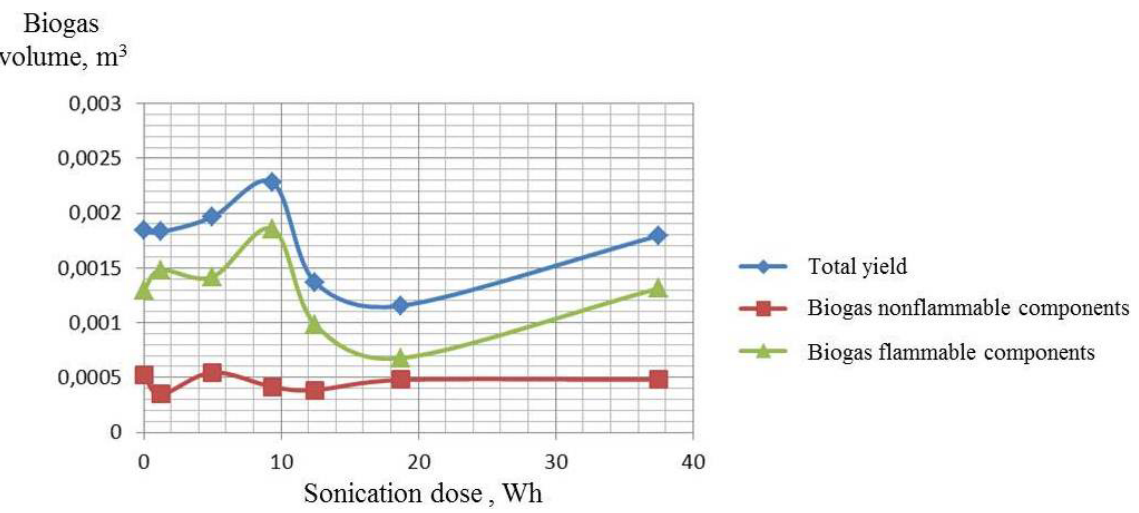

Figure 4: $\quad$ The biogas yield depending on the integral sonication dose.

\section{Conclusion}

Our investigations show that the sonication result in the biogas flammable components increase of $34-43 \%$. Sonication maximum efficiency is achieved at a moderate sonication dose. Additional energy costs for an ultrasound field with a maximum exposure time (half an hour) for the process total duration more than 20 days is less than $1 \%$.

\section{References}

[1] Eder, B. \& Shults, N. Biogas plants: ZorgBiogas, 2008.

[2] Zhang Hanjie. Sludge treatment to increase biogas production: Royal Institute of Technology TRITA-LWR Degree Project 10-20, SE-100 44 Stockholm, Sweden, 2010. 